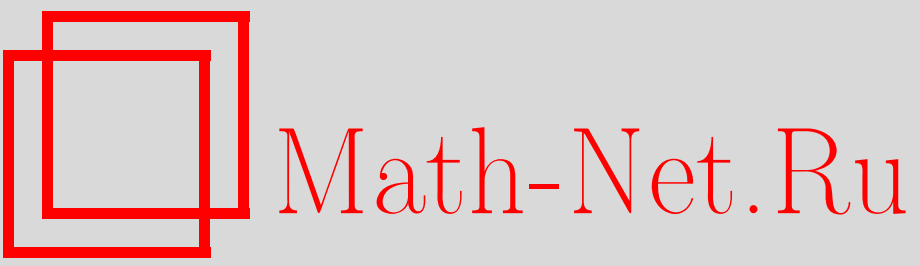

В. М. Нежинский, Псевдогомотопические инварианты сингулярных зацеплений, УМН, 1999, том 54, выпуск 3, 175-176

DOI: https://doi.org/10.4213/rm164

Использование Общероссийского математического портала Math-Net.Ru подразумевает, что вы прочитали и согласны с пользовательским соглашением

http://www . mathnet.ru/rus/agreement

Параметры загрузки:

IP: 18.208 .226 .222

26 апреля 2023 г., 15:37:09 


\section{ПСЕВДОГОМОТОПИЧЕСКИЕ ИНВАРИАНТЫ СИНГУЛЯРНЫХ ЗАЦЕПЛЕНИЙ}

\section{В. М. НЕЖинСКиЙ}

Предмет работы - сингулярные зацепления нескольких сфер размерности $k$ и одной сферы размерности $p$ в $(2 k+1)$-мерной сфере, где $k>1$. Пусть $t$ - число компонент сингулярного зацепления. В этой заметке для некоторых $t, k$ и $p$ построены полные наборы псевдогомотопических инвариантов таких сингулярных зацеплений и изучены свойства построенных инвариантов. Достойно внимания, что среди наших инвариантов имеются аналоги $\bar{\mu}$-инвариантов Милнора одномерных зацеплений. (Более подробно об этом написано в [1]; см. [1; Добавление].)

1. Формулировка проблемы. Пусть $r, k, p$ - натуральные числа. Сингулярньлм $(r, k, p)$-зацеплением называется последовательность непрерывных отображений $f_{1}: S^{k} \rightarrow$ $S^{2 k+1}, \ldots, f_{r}: S^{k} \rightarrow S^{2 k+1}, f: S^{p} \rightarrow S^{2 k+1}$ с попарно непересекающимися образами. Сингулярные $(r, k, p)$-зацепления $\left(f_{1}, \ldots, f_{r}, f\right)$ и $\left(g_{1}, \ldots, g_{r}, g\right)$ назьваются псевдогомотопными, если существует последовательность непрерывных отображений $F_{1}: S^{k} \times I \rightarrow S^{2 k+1} \times I$, $\ldots, F_{r}: S^{k} \times I \rightarrow S^{2 k+1} \times I, F: S^{p} \times I \rightarrow S^{2 k+1} \times I$ с попарно непересекающимися образами, такая, что $F_{i}(x, 0)=\left(f_{i}(x), 0\right), F_{i}(x, 1)=\left(g_{i}(x), 1\right), F(y, 0)=(f(y), 0)$ и $F(y, 1)=(g(y), 1)$ (для $1 \leqslant i \leqslant r, x \in S^{k}$ и $y \in S^{p}$ ). Наша проблема - это проблема псевдогомотопической классификации сингулярных $(r, k, p)$-зацеплений. Мы ограничимся рассмотрением только случая $k>1$.

2. Определения и свойства инвариантов. Пусть $L=\left(f_{1}, \ldots, f_{r}, f\right)$ - сингулярное $(r, k, p)$-зацепление.

Инварианты $\mu(i, j)(L)$. Пусть $1 \leqslant i \neq j \leqslant r$. Мы определяем $\mu(i, j)(L)$ как коэффицциент зацепления отображения $f_{i}$ с $f_{j}$. Хорошо известно, что $\mu(i, j)(L)=(-1)^{k+1} \mu(j, i)(L)$.

Назовем сингулярное $(r, k, p)$-зацепление $\left(g_{1}, \ldots, g_{r}, g\right)$ типичным, если $g_{1}, \ldots, g_{r}$ - гладкие вложения. (Нетрудно доказать, что любое сингулярное $(r, k, p)$-зацепление псевдогомотопно типичному.) Предположим, что сингулярное $(r, k, p)$-зацепление $L$ типично. Пусть $i \leqslant r$ - натуральное число. Положим $X=S^{2 k+1} \backslash \operatorname{Im} f_{i}$ и обозначим через $h$ вложение $S^{k} \rightarrow X$ такое, что коэффициент зацепления подмногообразия $h\left(S^{k}\right)$ сферы $S^{2 k+1}$ с $\operatorname{Im} f_{i}$ равен 1. Нетрудно проверить, что $h$ - гомотопическая эквивалентность. Пусть $h^{\prime}: X \rightarrow S^{k}$ - отображение, гомотопически обратное к $h$. Рассмотрим отображение $S^{p} \rightarrow S^{k}$, задаваемое формулой $x \mapsto h^{\prime}(f(x))$, и обозначим через $u$ его гомотопический класс. Мы полагаем $\mu(i, r+1)(L)=E u$ (где $E$ - гомоморфизм надстройки $\left.\pi_{p}\left(S^{k}\right) \rightarrow \pi_{p+1}\left(S^{k+1}\right)\right)$.

Инварианты $\nu(i, j, r+1)(L)$. Пусть $1 \leqslant i \neq j \leqslant r$. Рассмотрим какое-нибудь сохраняющее ориентацию гладкое вложение $g: D^{2 k+1} \rightarrow S^{2 k+1}$ такое, что $g(0) \in \operatorname{Im} f_{i}$ и $\operatorname{Im} g \cap$ $\operatorname{Im} f_{j}=\varnothing$. Возьмем какую-нибудь точку $x_{0} \in \operatorname{Im} f_{j}$ и выберем гомотопическую эквивалентность $h: S^{2 k+1} \backslash\left(g(0) \cup x_{0}\right) \rightarrow S^{2 k}$ такую, что $h(g(x))=x$ для $x \in S^{2 k}\left(=\partial D^{2 k+1}\right)$. Мы определяем $\nu(i, j, r+1)(L)$ как гомотопический класс отображения $S^{p} \rightarrow S^{2 k}$, задаваемого формулой $x \mapsto h(f(x))$.

Несложная проверка показьвает, что если $p<4 k-1$, mo $\nu(r, j, r+1)(L)=-\nu(j, r, r+1)(L)$ $u \nu(i, j, r+1)(L)=\nu(i, r, r+1)(L)-\nu(j, r, r+1)(L)$ для $i \neq r$.

Пусть $G$ - абелева группа и $\left\|a_{i j}\right\|$ - целочисленная квадратная матрица порядка $r$. Определим гомоморфизм $\varkappa\left(G,\left\|a_{i j}\right\|\right): \bigoplus^{r-1} G \rightarrow \bigoplus^{r(r-1) / 2} G$ формулой

$$
\begin{aligned}
\left(u_{1}, \ldots, u_{r-1}\right) \mapsto & \left(a_{12}\left(u_{1}-u_{2}\right), \ldots, a_{1 r-1}\left(u_{1}-u_{r-1}\right), a_{1 r} u_{1},\right. \\
& \left.a_{23}\left(u_{2}-u_{3}\right), \ldots, a_{2 r-1}\left(u_{2}-u_{r-1}\right), a_{2 r} u_{2}, \ldots, a_{r-1 r} u_{r-1}\right) .
\end{aligned}
$$

Работа выполнена при частичной поддержке Российского фонда фундаментальных исследований (грант № 98-01-00832). 
Оказывается, если $p<3 k-2$, mo $(\nu(1, r, r+1)(L), \ldots, \nu(r-1, r, r+1)(L)) \in \operatorname{Ker} \varkappa\left(\pi_{p}\left(S^{2 k}\right)\right.$, $\|\mu(i, j)(L)\|)$, где ми положили $\mu(i, i)(L)=0$.

Положим $B=\vee^{r} S^{k}$ и обозначим для $1 \leqslant i \leqslant r$ через in $_{i}$ стандартное вложение $S^{k} \rightarrow B$. Пусть $\left(g_{1}, \ldots, g_{r}, g\right)$ - типичное сингулярное $(r, k, p)$-зацепление. Положим $Y=S^{2 k+1} \backslash\left(\operatorname{Im} g_{1} \cup\right.$ $\left.\cdots \cup \operatorname{Im} g_{r}\right)$; назовем гладкое вложение $h: B \rightarrow Y m$-структурой сингулярного $(r, k, p)$-зацепления $\left(g_{1}, \ldots, g_{r}, g\right)$, если (при $\left.i=1, \ldots, r\right)$ композиция отображения $h \circ \mathrm{in}_{i}$ и включения $Y \hookrightarrow Y \cup \operatorname{Im} g_{i}$ гомотопна постоянному отображению и коэффициент зацепления многообразий $h\left(\operatorname{in}_{i}\left(S^{k}\right)\right)$ и $\operatorname{Im} g_{i}$ равен 1 . Типичное сингулярное $(r, k, p)$-зацепление $\left(g_{1}, \ldots, g_{r}, g\right)$ назьвается простым, если существует такая его $m$-структура $h$, что $\operatorname{Im} g \subset h(B)$. Оказывается, если $p<$ $3 k-2$, то сингулярное $(r, k, p)$-зачепление $L$ псевдогомотопно простому тогда и только тогда, когда $\nu(1, r, r+1)(L)=\cdots=\nu(r-1, r, r+1)(L)=0$.

Инвариант $\mathrm{M}(L)$. Пусть $p<3 k-2$. Предположим, что сингулярное $(r, k, p)$-зацепление $L$ просто. Выберем какую-нибудь его $m$-структуру $h$ такую, что $\operatorname{Im} f \subset h(B)$, и обозначим через $u$ гомотопический класс отображения $S^{p} \rightarrow B$, задаваемого формулой $x \mapsto h^{-1}(f(x))$. Согласно теореме Хилтона о гомотопических группах букета сфер и тому, что $p<3 k-2$, существуют элементы $\mu_{i} \in \pi_{p}\left(S^{k}\right)$, где $1 \leqslant i \leqslant r$, и $\mu(i, j, r+1) \in \pi_{p}\left(S^{2 k+1}\right)$, где $1 \leqslant i<j \leqslant r$, такие, что

$$
u=\sum_{1 \leqslant i \leqslant r} \operatorname{in}_{i} \circ \mu_{i}+\sum_{1 \leqslant i<j \leqslant r}\left[\operatorname{in}_{j}, \operatorname{in}_{i}\right] \circ \mu(i, j, r+1)
$$

(знак [ , ] обозначает умножение Уайтхеда). Мы определяем $\mathrm{M}(L)$ как класс смежности элемента $(\mu(1,2, r+1), \ldots, \mu(1, r, r+1), \mu(2,3, r+1), \ldots, \mu(r-1, r, r+1)) \in \bigoplus^{r(r-1) / 2} \pi_{p}\left(S^{2 k-1}\right)$ по подгруппе $\operatorname{Im} \varkappa\left(\pi_{p}\left(S^{2 k-1}\right),\|\mu(i, j)(L)\|\right)$. (Замечу, что $E \mu_{i}=\mu(i, r+1)(L)$.)

3. Основные теоремы. Пусть: $\left\|a_{i j}\right\|$ - целочисленная квадратная матрица порядка $r$ такая, что $a_{i j}=(-1)^{k+1} a_{j i}(1 \leqslant i \neq j \leqslant r) ; b_{1}, \ldots, b_{r} \in E \pi_{p}\left(S^{k}\right) ; c_{1}, \ldots, c_{r-1}$ - элементы группш $\pi_{p}\left(S^{2 k}\right)$ такие, что $\left(c_{1}, \ldots, c_{r-1}\right) \in \operatorname{Ker} \varkappa\left(\pi_{p}\left(S^{2 k}\right),\left\|a_{i j}\right\|\right) ; d \in \operatorname{Coker} \varkappa\left(\pi_{p}\left(S^{2 k-1}\right)\right.$, $\left.\left\|a_{i j}\right\|\right)$.

ТеОРема СУШеСтвОвАнИя. Если $p<3 k-2$, то: существует типичное сингулярное $(r, k, p)$-зачепление $L$ такое, что $\mu(i, j)(L)=a_{i j}$, әде $1 \leqslant i \neq j \leqslant r, \mu(i, r+1)(L)=b_{i}$, где $1 \leqslant i \leqslant r, u \nu(i, r, r+1)(L)=c_{i}$, где $1 \leqslant i \leqslant r-1 ;$ существует простое сингулярное $(r, k, p)$-зацепление $L^{\prime}$ такое, что $\mu(i, j)\left(L^{\prime}\right)=a_{i j}$, где $1 \leqslant i \neq j \leqslant r, \mu(i, r+1)\left(L^{\prime}\right)=b_{i}$, əде $1 \leqslant i \leqslant r, u \mathrm{M}\left(L^{\prime}\right)=d$.

Теорема ЕДИнСтвенности. Если $p<2 k-1(c k>1), p=2 k+4 c k>6$ или $r=1$ c $p \leqslant 3 k-2$, то типичные сингулярные $(r, k, p)$-зачепления $L$ и $L^{\prime}$ псевдогомотопнь тогда и только тогда, когда $\mu(i, j)(L)=\mu(i, j)\left(L^{\prime}\right)$, где $1 \leqslant i<j \leqslant r+1$. Если $p=2 k+3$ c $k>5$ или $p=2 k+11$ c $k>13$, то типичные сингулярные $(r, k, p)$-зацепления $L$ и $L^{\prime}$ псевдогомотопны тогда и только тогда, когда $\mu(i, j)(L)=\mu(i, j)\left(L^{\prime}\right)$, где $1 \leqslant i<j \leqslant$ $r+1, u \nu(i, r, r+1)(L)=\nu(i, r, r+1)\left(L^{\prime}\right)$, где $1 \leqslant i \leqslant r-1$. Если $p<3 k-2$, то простые сингулярные $(r, k, p)$-зацепления $L$ и $L^{\prime}$ псевдогомотопны тогда и только тогда, когда $\mu(i, j)(L)=\mu(i, j)\left(L^{\prime}\right)$, где $1 \leqslant i<j \leqslant r+1, u M(L)=M\left(L^{\prime}\right)$.

\section{СПИСОК ЛИТЕРАТУРЫ}

[1] Нежинский В. М. // Зап. научн. семин. ПОМИ. 1998. Т. 252. С. 175-190. 